\title{
Institucionalización del compromiso ambiental de las universidades colombianas ${ }^{1}$
}

\section{Institutionalization of the environmental commitment of colombian universities}

Recibido: 31 de mayo de 2016- Revisado: 24 de agosto de 2016 - Aceptado: 03 de marzo de 2017.

\author{
Orlando Sáenz Zapata ${ }^{2}$ \\ Ángela María Plata Rangel ${ }^{3}$ \\ María Teresa Holguín Aguirre \\ William Manuel Mora Penagos ${ }^{5}$ \\ Norka Blanco Portela ${ }^{6}$
}

\section{Resumen}

A través de este trabajo se pretende identificar aspectos de la institucionalización del compromiso ambiental en un grupo de universidades colombianas a partir de cinco ámbitos establecidos: gobierno, docencia, investigación, extensión y gestión. Para esto se aplicó una encuesta de 25 preguntas, planteadas a partir del proyecto RISU: "Definición de indicadores para la evaluación de las políticas de sustentabilidad en universidades latinoamericanas". El análisis, de carácter estadístico descriptivo, se elaboró con base en una escala entre 1 y 25 puntos, con cinco intervalos de igual magnitud para medir y clasificar el nivel de compromiso ambiental de las universidades. La encuesta fue respondida por 36 universidades del país. El nivel promedio hallado del compromiso de las IES colombianas es alto, con un valor de 18 puntos. Los ámbitos en los que el compromiso es más alto, son los de investigación y docencia, con un mismo valor promedio de 4,0 entre 5,0 posibles. Les siguen en su orden los de extensión $(3,6)$, gestión $(3,5)$ y gobierno $(3,3)$.

\section{Palabras clave}

Compromiso ambiental, educación superior, Universidad y ambiente.

\begin{abstract}
Through this work we intend to identify aspects of the institutionalization of environmental commitment in a group of Colombian universities from five established areas: government, teaching, research, extension and management. To this end, a survey of 25 questions was applied, based on the RISU project: "Definition of indicators for the evaluation of sustainability policies in Latin American universities". The analysis, of descriptive statistical nature, was elaborated based on a scale between 1 and 25 points, with five intervals of equal magnitude to measure and classify the environmental commitment level of the universities. The survey was answered by 36 universities in the country. The average level found in the commitment of the Colombian IES is high, with a value of 18 points. The areas in which the commitment is highest, are those of research and teaching, with an average value of 4.0 out of 5.0 possible. They are followed by extension (3.6), management (3.5) and government $(3,3)$.
\end{abstract}

\section{Keywords}

Environmental commitment, higher education, university and environment.
1 Artículo de investigación. Proyecto "Universidades sostenibles fase 1", coordinado desde la Universidad Sergio Arboleda, en cooperación con las Universidad de Ciencias Aplicadas y Ambientales, Universidad Libre de Colombia, Universidad Distrital Francisco José de Caldas y Universitaria Agustiniana.

${ }^{2}$ Docente investigador de la Universidad de Ciencias Aplicadas y Ambientales; Sociólogo de la Universidad Autónoma Latinoamericana y M. Sc. en Desarrollo Urbano de El Colegio de México; Estudiante de Doctorado de la Universidad Autónoma de Barcelona. Correo electrónico: osaenz@udca.edu.co ${ }^{3}$ Docente investigadora de la Universidad Sergio Arboleda; Ecóloga de la Pontifica Universidad Javeriana; M. Sc. en Ciencias Ambientales de la China University of Geosciences; Coordinadora de la Red Ambiental de Universidades Sostenibles; Correo electrónico: angela.plata@usa.edu.co.

${ }^{4}$ Docente Investigadora de la Universidad Libre de Colombia; Licenciada en Química y Biología de la Universidad Libre; Ph. D. (C) en Desarrollo Sustentable de la Universidad de Manizales. Correo electrónico: mariat.holguina@ unilibrebog.edu.co.

${ }^{5}$ Docente de carrera de la Universidad Distrital Francisco José de Caldas, Facultad del Medio Ambiente; Ph. D. en Educación Ambiental por la Universidad de Sevilla, España; Investigador de los grupos: DIDAQUIM y ALTERNACIENCIAS. Correo electrónico: wmora@udistrital.edu.co

${ }^{6}$ Docente e investigadora de la Universitaria Agustiniana. Bióloga de Universidad de la Habana; M. Sc. en Educación de la Universidad Externado de Colombia; Doctoranda en Investigación, Organización e Innovación, Correo electrónico: norka.blanco@ uniagustiniana.edu.co.

Para citar este artículo use: Sáenz, O. Plata, A., Holguín, A., Mora, W., \& Blanco, N. (2017). Institucionalización del compromiso ambiental de las universidades colombianas. Civilizar Ciencias Sociales y Humanas, 17(33), 189-208. doi:10.22518/16578953.908 


\section{Introducción}

Diferentes grupos de investigadores de América Latina y el Caribe, entre ellos el equipo Universidad Ambiente y Sustentabilidad, conformado por integrantes de las dos redes ambientales universitarias del país, han denominado "institucionalización del compromiso ambiental de las universidades" a la etapa más reciente del proceso que, desde la década de los setenta, se entendía como la "incorporación de la dimensión ambiental en la educación superior", y que en los años noventa algunos llamaron "ambientalización de las instituciones de educación superior (IES)".

Para quienes proponen la nueva denominación, la fase actual del proceso se diferencia de las anteriores porque en ellas la iniciativa de la inclusión de los temas ambientales en las IES estaba en manos de personas o colectivos de profesores, funcionarios o estudiantes con poco o ningún respaldo institucional; en cambio en los últimos años se exige, cada vez más, que sean las propias instituciones, representadas por sus más altas instancias directivas, las que asuman este compromiso (Equipo Universidad, Ambiente y Sustentabilidad -[UAS], 2014b).

Se entiende entonces que "incorporar" o "ambientalizar", son un nivel menos avanzado que "institucionalizar", ya que incorporar es algo parcial y ambientalizar viene de la traducción de la palabra "greening", que en países angloparlantes se enfoca a la creación de campus verdes, y aunque la ambientalización pueda ser de carácter institucional, no cubre todas las acciones de ambiente que se obtienen desde las funciones básicas de una Universidad: formación, investigación, extensión y proyección social.

Cuando se incorpora el concepto de "institucionalizar", se hace referencia a convertir algo en corporativo, es decir que genere compromisos desde el nivel directivo, permeando todos los espacios y por ende, todas las funcio- nes sustantivas de una institución de educación superior (IES).

Generalmente se cree que la incorporación de la dimensión ambiental en la educación superior se ha desarrollado en las últimas cuatro décadas, pero en una investigación histórica realizada por Sáenz (2012), sobre las primeras etapas de este proceso, se demostró que sus orígenes se remontan en Colombia a los comienzos de la segunda mitad del siglo pasado. Puesto que en los años cincuenta todavía no se había construido socialmente el actual concepto de Ambiente, los primeros programas de formación superior, que ahora se consideran como ambientales, se referían a la conservación de la naturaleza y al aprovechamiento de los recursos naturales renovables.

\section{Antecedentes de investigación}

El interés por conocer los avances en el proceso de incorporación de la dimensión ambiental en las universidades de la región comenzó en la segunda mitad de la década de los setenta y desde entonces se ha mantenido e incluso, aumentado. Su principal motivación ha sido siempre la misma: contar con el conocimiento necesario para orientar e intervenir sobre este proceso de la manera más acertada posible.

Para entender la lógica de esta causa, se debe recordar que durante sus primeras etapas estuvo centrada, principalmente, en la "formación ambiental superior" y específicamente en el diseño y ofrecimiento de nuevos programas de pregrado o posgrado, relacionados directa o indirectamente con el ambiente, en las más diversas acepciones de este concepto. Según la periodización propuesta por Sáenz (2012), en Colombia se pueden distinguir tres grandes etapas del proceso de incorporación de la dimensión ambiental en la educación superior, definidas desde la perspectiva de la formación profesional y de posgrado: la primera etapa se concentró en programas de "educación relativa 
a la naturaleza y a los recursos naturales" y se desarrolló entre 1950 y 1970; la siguiente se ocupó de la "educación ambiental propiamente dicha" y se ubica entre 1971 y 1990;.1a última y actual fase, hace más énfasis en la "educación para el desarrollo sostenible o educación para la sostenibilidad" y está en curso desde 1991 (p.184).

Sin tratar de presentar un relato exhaustivo de esta historia, vale la pena destacar algunos de los eventos más importantes. La primera institución que manifestó interés en conocer los avances en la incorporación de la dimensión ambiental en la educación superior, fue el Centro Internacional de Formación en Ciencias Ambientales (CIFCA), establecimiento creado en 1973 por una cooperación entre el gobierno español y el Programa de las Naciones Unidas para el Medio Ambiente (PNUMA). En 1977, el CIFCA realizó el primer diagnóstico, titulado "Panorama de los Estudios Superiores Medioambientales en América Latina” (CIFCA, 1978).

En los ochenta, el PNUMA continuó haciendo un seguimiento a los avances en este proceso y para ello contó con el apoyo de la Organización de las Naciones Unidas para la Educación, la Ciencia y la Cultura (UNESCO). Así, entre 1984 y 1985, ambas agencias de la ONU adelantaron el "Diagnóstico de la Incorporación de la Dimensión Ambiental en los Estudios Superiores en América Latina y el Caribe" (UNESCO, PNUMA e ICFES, 1988). Dicho estudio fue coordinado por el colombiano Augusto Ángel Maya y recopiló información sobre 166 universidades de más de 20 países latinoamericanos. Aunque su énfasis siguió siendo el de los programas de pregrado y posgrado, también se obtuvo información sobre investigación, extensión y aspectos administrativos relacionados con el ambiente.

El Colegio de México, institución pública y de carácter universitario, mantiene también esta línea y presenta en 1984, el primer diagnóstico nacional de la región: el "Estudio sobre la incorporación de la Formación Ambiental a la Educación Superior en México" (Sánchez, 1984), con el cual "se pretendió hacer un diagnóstico de la situación en materia de formación ambiental que guardaban los estudios superiores de escuelas públicas y privadas, así como identificar los programas más propicios para incorporarles contenidos ambientales" (Bravo, 2005, p. 5).

En este artículo, dadas las características de espacio concedido, no se dará detallada cuenta de los numerosos diagnósticos, análisis, estudios, investigaciones, reportes y otros tipos de documentos que se han ocupado del proceso de incorporación de la dimensión ambiental en la educación superior, y que podríamos organizar en dos categorías, según la escala territorial de referencia: casi una decena son estudios en el marco regional latinoamericano y del caribe, y casi medio centenar son análisis con una cobertura nacional. Estos últimos se reseñaron con detalle en el artículo "Diagnósticos Nacionales sobre la Inclusión de Consideraciones Ambientales en las Universidades de América Latina y el Caribe" (Sáenz, 2015a). El primer grupo de documentos será presentado y comentado en el artículo, que actualmente se redacta, "Diagnósticos Regionales sobre la Inclusión de Consideraciones Ambientales en las Universidades de América Latina y el Caribe" (Sáenz, en prensa).

El estudio regional más reciente es "Definición de Indicadores para la evaluación de las políticas de sustentabilidad en universidades latinoamericanas" (Benayas et al., 2014), realizado durante 2013 y 2014 por la Red de Indicadores de Sostenibilidad en las Universidades (RISU), de la que hacen parte los autores de este artículo. La Universidad Autónoma de Madrid (UAM) lo coordinó y contó con la participación de 65 universidades de 10 países de América Latina y el Caribe, entre ellas cinco colombianas.

La red RISU hace parte de la Alianza de Redes Iberoamericanas de Universidades por 
la Sustentabilidad y el Ambiente (ARIUSA), que agrupa actualmente 22 redes universitarias ambientales, lo que corresponde a más de 369 universidades en 15 países de Iberoamérica. En diciembre de 2013 ARIUSA organizó el Primer Foro Latinoamericano de Universidades y Sostenibilidad, que tuvo lugar en Viña del Mar (Chile). Sáenz (2015b), señala que las dos principales recomendaciones de este Foro fueron: a) la "construcción de un sistema de indicadores de la sostenibilidad en las Universidades, adecuado a las condiciones particulares de las universidades de la región", y b) la "realización de un diagnóstico de la situación actual del proceso de ambientalización de las instituciones de educación superior a escala regional y en la mayoría de los países latinoamericanos y caribeños" (p. 127). El proyecto RISU respondió directamente a la primera de estas recomendaciones y como resultado, se propusieron 114 preguntas, organizadas en 11 dimensiones (Benayas et al., 2014).

Además de que las universidades colombianas han participado en todos los diagnósticos o estudios regionales que se han elaborado desde mediados de la década de los setenta sobre la incorporación de la dimensión ambiental en la educación superior, Colombia es uno de los países latinoamericanos con mayor número de diagnósticos o estudios nacionales sobre este proceso. Hasta 2015 se tenían identificados 40 trabajos de distinto tipo para 11 países latinoamericanos y caribeños. Entre ellos, México contaba con diez, Colombia con nueve, Brasil con seis, Venezuela con cinco y Perú con tres. (Sáenz, 2015a).

Los nueve trabajos en Colombia constituyen los antecedentes directos de esta investigación y aunque por obvias razones no se reproducen los textos correspondientes, sí vamos a destacar aquéllos con los que se relaciona más directamente este estudio, ya sea porque son un referente obligado o porque se coincide parcialmente en el enfoque teórico o en la metodología.
Indiscutiblemente, la referencia más importante de la presente publicación, es el "Diagnóstico de los Programas Ambientales Universitarios en Colombia", elaborado en 1987 por Augusto Ángel Maya, con el apoyo de Instituto Colombiano para la Evaluación de la Educación (ICFES), el Instituto Nacional de los Recursos Naturales Renovables y del Ambiente (INDERENA) ${ }^{1}$ y la Universidad Nacional de Colombia (UNAL). Ángel retomó y amplió el enfoque y la metodología que había utilizado en el diagnóstico regional del PNUMA de 1984, mencionado anteriormente; también utilizó la información específica que habían aportado las IES colombianas para el estudio regional y la complementó con una nueva encuesta y la revisión de varias fuentes de divulgación. En total, este diagnóstico proporciona información sobre 70 instituciones nacionales de educación superior, entre las cuales se cuentan casi todas las que respondieron en 2014 la encuesta con la que se recopilaron los insumos y datos para el presente análisis.

Por otra parte, el antecedente más reciente y de directa influencia en este trabajo, es el estudio que realizó, durante 2007 y 2008, un equipo interinstitucional de investigación en el que colaboraron tres de los autores de este artículo; cuyos objetivos principales eran hacer el "diagnóstico de base sobre la inclusión y dinamización de la dimensión ambiental, a partir del análisis de las experiencias con las universidades seleccionadas" y elaborar una "guía técnica para la formulación, implementación y seguimiento de un modelo para la inclusión de la dimensión ambiental en la educación superior en el Distrito Capital". Fue coordinado por la Universidad Piloto de Colombia y participaron investigadores de las siguientes universidades: Universidad de Ciencias Aplicadas y Ambientales, Universidad Distrital, Universidad Libre, Universidad Nacional de Colombia, Universidad Pedagógica Nacional y la Institución Universitaria Politécnico Grancolombiano; en 2011 la Unión de Universidades de América Latina y el Caribe (UDUAL), le otorgó el Premio "Andrés Bello" a la me- 
jor investigación sobre educación superior en América Latina y el Caribe.

Los resultados de dicho estudio los presentó recientemente la profesora Yenny Constanza Román Núñez en el libro "Sistema Ambiental Universitario: Modelo integrado de Gestión para la Inclusión de la Dimensión Ambiental y Urbana en la Educación Superior" (Román, 2016).

Román estructuró en su libro la noción de "Sistema Ambiental Universitario", con el que coinciden varios de los autores de este artículo desde su participación en el estudio interinstitucional antes mencionado. Los desarrollos más recientes de este concepto, por parte del actual equipo de investigación, han llevado a ampliar su alcance y a denominarlo "Sistema Ambiental Institucional" para la educación superior; así denominado, es uno de los fundamentos del enfoque teórico que orienta la investigación y que se presentará de manera más amplia en un libro en preparación.

Como se puede ver en esta presentación de antecedentes, desde 1985 hasta ahora, la reflexión y la investigación sobre los avances en la ambientalización de las instituciones colombianas de educación superior siempre han estado acompañando el desarrollo de este proceso. Del estudio de la etapa actual, que se describe como "institucionalización del compromiso ambiental en la educación superior", se ocupa el Equipo Universidad, Ambiente y Sustentabilidad (UAS), un nuevo equipo interinstitucional de investigación que integran los autores de este artículo.

A nivel regional y desde 1977, el interés es el mismo y esto se manifestó, a un alto nivel político en 2014, en el XIX Foro de Ministros del Ambiente en América Latina y el Caribe. En esta reunión, los Ministros decidieron nuevamente "desarrollar un diagnóstico por país sobre la inclusión de consideraciones ambientales en las universidades (en cuatro ámbitos que abarcan el currículum, la gestión institucional, la extensión y la investigación), con base, tanto en indicadores comunes, como diferenciados por país" (PNUMA, 2014).

\section{Objetivo general}

En cumplimiento de su misión fundamental y de sus objetivos compartidos, la Red Colombiana de Formación Ambiental (RCFA) y la Red Ambiental de Universidades Sostenibles (RAUS) decidieron producir conjuntamente y para Colombia, el diagnóstico sobre la inclusión de consideraciones ambientales en las universidades, planteado en 2014 por el Foro de Ministros del Ambiente de América Latina y el Caribe. De esta manera se le da continuidad a una tarea colectiva, como la que se registra en los antecedentes reseñados, para mantener actualizada la información y el saber sobre los avances de dicho proceso.

En consecuencia, el principal objetivo de este análisis era identificar aspectos de la institucionalización del compromiso ambiental en un grupo de universidades colombianas a partir de cinco ámbitos establecidos: gobierno, docencia, investigación, extensión y gestión.

Este trabajo fue asumido por el Equipo Universidad, Ambiente y Sustentabilidad (UAS), conformado por investigadores de la Universidad Sergio Arboleda, Universidad Libre, Universidad Distrital, Universitaria Agustiniana y la Universidad de Ciencias Aplicadas y Ambientales.

\section{Materiales y Métodos}

Esta investigación aplica un alcance de tipo exploratorio, que según Hernández (2014):

Sirven para familiarizarnos con fenómenos relativamente desconocidos, obtener información sobre la posibilidad de llevar a cabo una investigación más completa respecto de un contexto particular, indagar nuevos pro- 
blemas, identificar conceptos o variables promisorias, establecer prioridades para investigaciones futuras, o sugerir afirmaciones y postulados (p.91).

Con este trabajo, se busca identificar aspectos de la institucionalización del compromiso ambiental en un grupo de universidades colombianas a partir de cinco ámbitos establecidos: gobierno, docencia, investigación, extensión y gestión.

\section{Herramienta de recolección y ámbitos de evaluación.}

Para la recolección de los datos en las Universidades (47 Instituciones de Educación Superior, de las cuales 36 se encuentran acreditadas como Universidades), se diseñó una herramienta con un total de 25 preguntas; dichas preguntas provienen del proyecto "Definición de Indicadores para la evaluación de las políticas de sustentabilidad en universidades latinoamericanas", de la red RISU, el cual se tomó como referente Latinoamericano para este trabajo; en él participaron once redes universitarias ambientales y 65 universidades de 10 países latinoamericanos, entre ellas cinco universidades colombianas, de las cuales cuatro integran el Equipo UAS.

En el estudio adelantado por RISU se formularon un total de 114 preguntas, las cuales estaban organizadas en 11 "temáticas" o "dimensiones": Política de sostenibilidad; Sensibilización y participación; Responsabilidad socioambiental; Docencia; Investigación y transferencia; Urbanismo y biodiversidad; Energía; Agua; Movilidad; Residuos, y Contratación responsable.

Para el diseño de la herramienta del presente estudio y con base en la clasificación de funciones y actividades de las instituciones de educación superior que habitualmente se utiliza en América Latina y que se expresan en la decisión del Foro de Ministros del Ambiente de los países de la región, el equipo UAS decidió reorganizar estas once "temáticas" o "dimensiones" en cinco dominios, considerados como los "ámbitos" de acción a observar,

Cabe señalar que la reagrupación propuesta se basa en la consideración de que los criterios 6 al 11 del proyecto de la Red RISU (Urbanismo y biodiversidad; Energía; Agua; Movilidad; Residuos, y Contratación responsable), se podrían clasificar bajo un solo ámbito, designado como "Gestión y ordenamiento ambiental del campus", ya que dichas temáticas hacen referencia a la gestión ambiental institucional dentro de los campus universitarios y corresponden a lo que generalmente se agrupa bajo los Sistemas de Gestión Ambiental-SGA.

Por consiguiente, se definieron los siguientes cinco ámbitos de acción institucional de las universidades en lo relativo a sus compromisos con el ambiente y la sustentabilidad: a) Gobierno y participación; b) Docencia y formación; c) Investigación y tecnología; d) Extensión o proyección; e) Gestión y ordenamiento. En adelante, para facilitar la lectura y la presentación en gráficas, se designarán también con la primera palabra de cada etiqueta.

Para cada uno de estos cinco ámbitos de acción institucional se retomaron cinco preguntas del cuestionario del Proyecto RISU, las cuales se seleccionaron dado que eran preguntas básicas que permitían conocer el nivel de institucionalización en las universidades. Como resultado, esta nueva encuesta, que se considera esencial para la evaluación del compromiso ambiental de las instituciones de educación superior en Colombia, consiste en una serie de 25 preguntas.

\section{Características de la encuesta.}

Una novedad importante en las encuestas del Proyecto RISU y del Equipo UAS, al compararlas con estudios similares anteriores realizados tanto en Colombia como en otros países latinoamericanos, es su énfasis en la indagación sobre las políticas institucionales de las univer- 
sidades con respecto a los temas de ambiente y sustentabilidad. Aunque algunos de los diagnósticos y estudios previos se basaron en información oficial proporcionada por las instituciones de educación superior, las preguntas de sus encuestas se orientaban principalmente hacia sus programas, proyectos y actividades. Por el contrario, en los nuevos formularios el énfasis se centra en las orientaciones y decisiones políticas que se establecen desde el más alto nivel institucional.

En resumen, para la encuesta en Colombia, la gran mayoría de las 25 preguntas tomadas del formulario del Proyecto RISU conducen a obtener información sobre decisiones y acciones institucionales de cada universidad que expresen su compromiso con el ambiente y la sustentabilidad. Sólo unas pocas preguntas se refieren a programas, proyectos o acciones específicas en el campo ambiental.
Una vez diseñada, la encuesta se subió a Google Docs para que fuera respondida en línea y se puso a disposición de todas las universidades colombianas y otras instituciones de educación superior interesadas en su evaluación,

En la presentación de la encuesta se hizo énfasis en que la participación de las universidades en este estudio es institucional, por lo tanto, para responder las preguntas se debía contar con la autorización y respaldo de sus directivos. Preferiblemente, las respuestas institucionales deberían ser aportadas por el equipo de trabajo o por el funcionario responsable de los asuntos ambientales de la universidad.

En la Tabla 1 se presentan los cinco ámbitos de acción y las 25 preguntas básicas de la encuesta que se utilizó como instrumento de recolección de la información que sirve de base al presente estudio.

\section{Tabla 1. Encuesta para el diagnóstico de la Institucionalización del compromiso ambiental de las universidades colombianas}

\begin{tabular}{|c|c|}
\hline $\begin{array}{l}\text { AMBITO DE } \\
\text { ACCIÓN }\end{array}$ & PREGUNTAS BÁSICAS \\
\hline \multirow{5}{*}{$\begin{array}{l}\text { GOBIERNO Y } \\
\text { PARTICIPACIÓN } \\
\text { AMBIENTAL }\end{array}$} & $\begin{array}{l}\text { 1. ¿Existe un documento que defina la política que la Universidad desarrolla en el ámbito de la } \\
\text { sustentabilidad o el ambiente? }\end{array}$ \\
\hline & $\begin{array}{l}\text { 2. ¿Existe una unidad, oficina o servicio de carácter técnico-administrativo con dedicación exclusiva para } \\
\text { los temas de sustentabilidad ambiental? }\end{array}$ \\
\hline & $\begin{array}{l}\text { 3. La organización de la acción por la sustentabilidad ambiental en su Universidad corresponde a: a) Un } \\
\text { conjunto de acciones o agenda; b) Un Plan de Acción Ambiental; c) Un Sistema Ambiental Institucional }\end{array}$ \\
\hline & $\begin{array}{l}\text { 4. ¿La unidad, oficina o servicio de sustentabilidad ambiental dispone de presupuesto propio para realizar } \\
\text { actividades o inversiones en esta materia? }\end{array}$ \\
\hline & $\begin{array}{l}\text { 5. ¿Están involucrados los docentes, estudiantes o administrativos en el diseño e implementación de la } \\
\text { política ambiental o de sustentabilidad en la Universidad? }\end{array}$ \\
\hline \multirow{5}{*}{$\begin{array}{l}\text { DOCENCIA } \\
\text { Y FORMACIÓN } \\
\text { AMBIENTAL }\end{array}$} & $\begin{array}{l}\text { 6. ¿Existe en la política ambiental o de sustentabilidad de la Universidad mención expresa a las actividades } \\
\text { docentes o de formación? }\end{array}$ \\
\hline & $\begin{array}{l}\text { 7. ¿Se incorpora en algunos de los currículos académicos de las distintas carreras la perspectiva de la } \\
\text { sustentabilidad ambiental? }\end{array}$ \\
\hline & $\begin{array}{l}\text { 8. ¿Se han definido de manera explícita competencias o capacidades transversales básicas en sustentabilidad } \\
\text { ambiental en algunas carreras que ofrece la Universidad? }\end{array}$ \\
\hline & $\begin{array}{l}\text { 9. ¿Existen en la Universidad programas de grado o pregrado (carreras profesionales) específicos sobre } \\
\text { ambiente o sustentabilidad? }\end{array}$ \\
\hline & $\begin{array}{l}\text { 10. ¿Existen en la Universidad programas de posgrado (Especializaciones, Maestrías o Doctorados) } \\
\text { específicos sobre ambiente o sustentabilidad? }\end{array}$ \\
\hline
\end{tabular}




\begin{tabular}{|c|c|}
\hline $\begin{array}{l}\text { ÁMBITO DE } \\
\text { ACCIÓN }\end{array}$ & PREGUNTAS BÁSICAS \\
\hline \multirow{5}{*}{$\begin{array}{c}\text { INVESTIGACIÓN } \\
\text { Y TECNOLOGÍA } \\
\text { AMBIENTAL }\end{array}$} & $\begin{array}{l}\text { 11. ¿Existe en la política ambiental o de sustentabilidad de la Universidad mención expresa a la } \\
\text { investigación y transferencia de tecnología? }\end{array}$ \\
\hline & $\begin{array}{l}\text { 12. ¿Existe en la Universidad algún grupo de investigación específico sobre ambiente o sustentabilidad } \\
\text { formalmente constituido? }\end{array}$ \\
\hline & $\begin{array}{l}\text { 13. ¿La Universidad ha participado en proyectos nacionales o internacionales de investigación sobre } \\
\text { ambiente o sustentabilidad? }\end{array}$ \\
\hline & $\begin{array}{l}\text { 14. ¿Se consideran criterios de sustentabilidad y evaluación del impacto ambiental en la aprobación, por } \\
\text { parte de la Universidad, de los proyectos de investigación y transferencia de tecnología? }\end{array}$ \\
\hline & $\begin{array}{l}\text { 15. ¿Existen estrategias para incentivar la utilización de la Universidad y de sus campus o instalaciones en } \\
\text { la realización de investigaciones sobre temas ambientales y de sustentabilidad? }\end{array}$ \\
\hline \multirow{5}{*}{$\begin{array}{l}\text { EXTENSIÓN O } \\
\text { PROYECCIÓN } \\
\text { AMBIENTAL }\end{array}$} & $\begin{array}{l}\text { 16. ¿Se implementa un plan específico, eje estratégico o plan de acción de responsabilidad socioambiental } \\
\text { que ha sido aprobado por algún órgano de gobierno de la Universidad? }\end{array}$ \\
\hline & $\begin{array}{l}\text { 17. ¿Existen programas permanentes de intervención para contribuir a la solución de problemas } \\
\text { ambientales o de sustentabilidad en comunidades? }\end{array}$ \\
\hline & $\begin{array}{l}\text { 18. ¿Existen programas permanentes de colaboración con gobiernos (locales, regionales o nacionales) } \\
\text { para la formulación o implementación de políticas públicas ambientales o de sustentabilidad? }\end{array}$ \\
\hline & $\begin{array}{l}\text { 19. ¿Existen programas permanentes de vinculación con el sector empresarial del entorno en materia de } \\
\text { ambiente o sustentabilidad? }\end{array}$ \\
\hline & $\begin{array}{l}\text { 20. ¿Participa la Universidad en redes universitarias nacionales o internacionales por el ambiente o la } \\
\text { sustentabilidad }\end{array}$ \\
\hline \multirow{5}{*}{$\begin{array}{l}\text { GESTIÓN Y } \\
\text { ORDENAMIENTO } \\
\text { AMBIENTAL }\end{array}$} & $\begin{array}{l}\text { 21. ¿Se implementa un plan específico, eje estratégico o línea de acción de sustentabilidad ambiental que } \\
\text { incluya aspectos de ahorro en agua, sanidad para consumo humano, riego y gestión de aguas residuales? }\end{array}$ \\
\hline & $\begin{array}{l}\text { 22. ¿Se implementa un plan específico, eje estratégico o línea de acción de sustentabilidad ambiental que } \\
\text { incluya aspectos relacionados con alumbrado, climatización y energías renovables? }\end{array}$ \\
\hline & $\begin{array}{l}\text { 23. ¿Se implementa un plan específico, eje estratégico o línea de acción de sustentabilidad ambiental para } \\
\text { la minimización, recogida selectiva y manejo adecuado de los residuos sólidos? }\end{array}$ \\
\hline & $\begin{array}{l}\text { 24. ¿Se implementa un plan específico, eje estratégico o línea de acción de sustentabilidad o ambiental } \\
\text { sobre la gestión de las zonas verdes y la biodiversidad? }\end{array}$ \\
\hline & 25. ¿La planificación urbanística de la Universidad incluye criterios de sustentabilidad o ambientales? \\
\hline
\end{tabular}

Fuente: Elaboración propia.

Hay que señalar que las respuestas a 24 de las 25 preguntas de la encuesta se limitaron a un SI o un NO, a pesar de que en cada una de ellas se solicitó aportar información o documentos que respaldaran las respuestas positivas, e indicar la página web en la que se podrían encontrar.

\section{Escalas de medición.}

Se construyó una escala específica para cada uno de los cinco ámbitos de acción que comprende la encuesta (gobierno, docencia, investigación, extensión y gestión) y una escala 
general que resulta de la suma de puntajes en todas las anteriores.

Al procesar las respuestas de cada Universidad, se le asigna un punto por cada respuesta positiva a las preguntas básicas. Entonces, el máximo puntaje posible para una universidad es de 5 en cada uno de los ámbitos de acción y de 25 para el total de la encuesta.

Tanto las escalas específicas como la general tienen los siguientes cinco niveles posibles, en su orden y del inferior al superior, así: muy bajo, bajo, medio, alto y muy alto. En las escalas específicas para cada ámbito de acción, estos niveles de compromiso ambiental de las instituciones de educación superior corresponden a los siguientes puntajes:

Tabla 2. Puntajes de los niveles de compromiso ambiental en cada ámbito de acción

\begin{tabular}{|l|c|}
\hline \multicolumn{1}{|c|}{ Niveles } & Puntajes \\
\hline Muy bajo & $0,0-1,0$ \\
\hline Bajo & $1,1-2,0$ \\
\hline Medio & $2,1-3,0$ \\
\hline Alto & $3,1-4,0$ \\
\hline Muy alto & $4,1-5,0$ \\
\hline
\end{tabular}

Fuente: Elaboración propia.

Por su parte, en la escala que mide el avance general en la institucionalización del compromiso ambiental de las universidades, los niveles se cuantifican con intervalos de igual valor que comprenden los siguientes rangos de puntaje:

Tabla 3. Puntajes de los niveles de compromiso ambiental en la escala general

\begin{tabular}{|l|c|}
\hline \multicolumn{1}{|c|}{ Niveles } & Puntajes \\
\hline Muy bajo & $1-5$ \\
\hline Bajo & $6-10$ \\
\hline Medio & $11-15$ \\
\hline Alto & $16-20$ \\
\hline Muy alto & $21-25$ \\
\hline
\end{tabular}

Fuente: Elaboración propia.

\section{Población y muestra.}

Desde el título del proyecto original se indicaba claramente el interés de esta investigación por conocer el compromiso ambiental de las universidades colombianas y esto implicaba dejar de lado a otras instituciones de educación superior (IES). Según datos del Ministerio de Educación Nacional (MEN), el país contaba en el año 2014 con un total de 288 instituciones de educación superior, de las cuales 82 eran Universidades y 206 otras IES (MEN, 2014).

Si bien es conocido que en los estudios que emplean encuestas es muy difícil cubrir la totalidad de las Universidades, en este proyecto se realizó un importante esfuerzo para lograrlo: a través de la Asociación Colombiana de Universidades (ASCUN) se envió una comunicación a la mayoría de los rectores, solicitándoles el diligenciamiento del formulario en línea. Este requerimiento se les reiteró por medio de los representantes de las universidades que integran la Red Colombiana de Formación Ambiental (RCFA) y la Red Ambiental de Universidades Sostenibles (RAUS).

Durante un periodo de cinco meses, entre agosto y diciembre de 2014, la encuesta fue respondida por 47 instituciones de educación superior de diferentes regiones del país. Estas fueron diligenciadas en su mayoría, por funcionarios responsables del área, oficina o departamento encargado de temas de ambiente y sustentabilidad; sin embargo, en muchos casos fueron los docentes de temas ambientales los que buscaron la información en varias dependencias de la institución y quienes finalmente resolvieron la encuesta.

De las 47 IES que participaron, 36 son reconocidas oficialmente como Universidades; la información que estas últimas proporcionaron, es la base de los resultados que ahora se presentan. El listado (Tabla 4), se ofrece de acuerdo con el orden de llegada de la encuesta diligenciada. 
Tabla 4. Universidades colombianas que respondieron la encuesta en 2014

\begin{tabular}{|c|c|}
\hline Universidades & Ciudades \\
\hline $\begin{array}{l}\text { Universidad de Ciencias Aplicadas y } \\
\text { Ambientales }\end{array}$ & Bogotá \\
\hline Universidad Autónoma de Manizales & Manizales \\
\hline Universidad de Caldas & Manizales \\
\hline Universidad Autónoma de Occidente & Cali \\
\hline Universidad El Bosque & Bogotá \\
\hline Universidad Simón Bolívar & Barranquilla \\
\hline Universidad Católica de Pereira & Pereira \\
\hline Universidad Católica de Colombia & Bogotá \\
\hline Pontificia Universidad Javeriana & Bogotá \\
\hline Universidad del Rosario & Bogotá \\
\hline Universidad de La Sabana & Chía \\
\hline $\begin{array}{l}\text { Universidad de Bogotá Jorge Tadeo } \\
\text { Lozano }\end{array}$ & Bogotá \\
\hline Universidad del Quindío & Armenia \\
\hline Universidad Industrial de Santander & Bucaramanga \\
\hline $\begin{array}{l}\text { Universidad Distrital Francisco José de } \\
\text { Caldas }\end{array}$ & Bogotá \\
\hline Universidad Pedagógica Nacional & Bogotá \\
\hline Universidad Central & Bogotá \\
\hline Universidad del Tolima & Ibagué \\
\hline Universidad Sergio Arboleda & Bogotá \\
\hline Universidad de Manizales & Manizales \\
\hline Universidad Santo Tomás & Bogotá \\
\hline Universidad de Pamplona & Pamplona \\
\hline Universidad Libre & Bogotá \\
\hline Universidad de La Salle & Bogotá \\
\hline Universidad Antonio Nariño & Bogotá \\
\hline Universidad Manuela Beltrán & Bogotá \\
\hline Universidad de Cartagena & Cartagena \\
\hline Universidad de los Llanos & Villavicencio \\
\hline Universidad del Valle & Cali \\
\hline Universidad de Medellín & Medellín \\
\hline Universidad de Cundinamarca & Fusagasugá \\
\hline Universidad Tecnológica del Chocó & Quibdó \\
\hline Universidad Militar Nueva Granada & Bogotá \\
\hline
\end{tabular}

\begin{tabular}{|l|l|}
\hline \multicolumn{1}{|c|}{ Universidades } & \multicolumn{1}{c|}{ Ciudades } \\
\hline Universidad de Antioquia & Medellín \\
\hline Universidad Tecnológica de Pereira & Pereira \\
\hline Universidad La Gran Colombia & Armenia \\
\hline
\end{tabular}

Fuente: Elaboración propia.

Recordemos que en el 2014, existen 82 Universidades reconocidas por el MEN; de acuerdo con la finalidad del presente estudio, se establece una muestra bajo un criterio por conveniencia, la cual es una técnica de muestreo no probabilístico donde los sujetos son seleccionados dada la conveniente accesibilidad de estos para el investigador. Es decir, la encuesta fue diligenciada por las Universidades que quisieron hacer su aporte a la investigación.

Para el análisis de los datos se recurrió a la estadística descriptiva con la cual, se interpretan los datos y son presentados en gráficas, cuadros y resúmenes. Para las respuestas de las Universidades, se empleó el análisis de frecuencias, identificando la media para el puntaje total de las instituciones y para cada uno de los cinco ámbitos, teniendo en cuenta puntajes mínimos y máximos.

\section{Resultados y Discusión}

El primer y más importante resultado de esta investigación es el alto nivel de institucionalización del compromiso ambiental en el grupo de Universidades colombianas que respondieron la encuesta básica. De un valor máximo posible de 25 puntos, la media para el conjunto de respuestas fue de 18 puntos, ubicado en el nivel alto. La mediana es 20, lo que significa que la mitad de las Universidades participantes están en el nivel alto o muy alto. La moda es 23, con seis instituciones que se clasifican en el nivel muy alto. El rango de los datos es de 17, resultado de la diferencia entre 25 y 8 , como puntajes máximo y mínimo obtenidos. La desviación estándar es de 5 . 
Gráfico 1. Puntaje general de compromiso ambiental de 36 Universidades colombianas, 2014

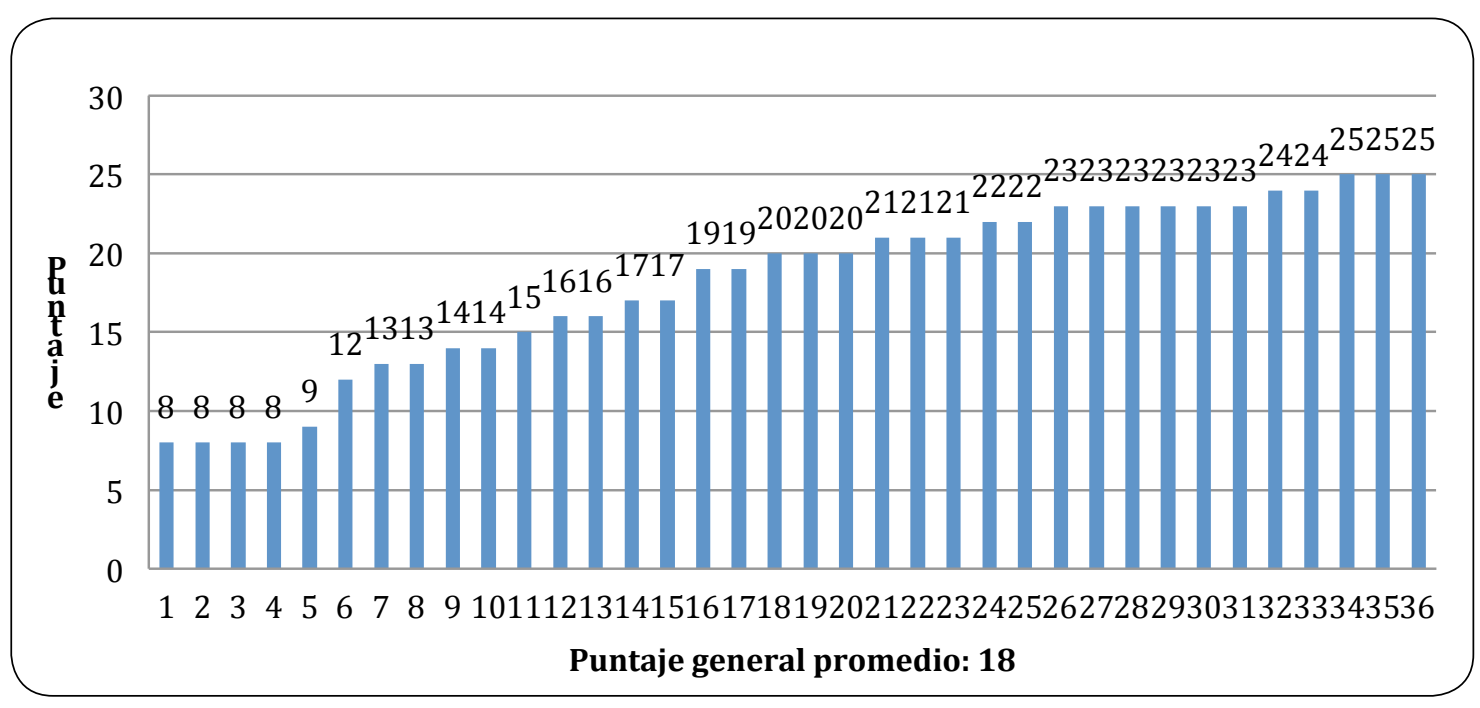

Fuente: Elaboración propia.

Como se aprecia en el Gráfico 1, el menor puntaje alcanzado por cuatro Universidades fue de 8. Por otra parte, fueron tres las instituciones que alcanzaron el máximo puntaje posible, pues respondieron positivamente todas las preguntas de la encuesta.
La tendencia general que se aprecia en el Grafico 1, es un alto nivel de institucionalización del compromiso ambiental de las Universidades colombianas. Esto se hace aún más evidente en el Gráfico 2, que presenta el número de estas en cada nivel, definido para la escala general de medición.

Gráfico 2. Número de Universidades colombianas por nivel de institucionalización de su compromiso ambiental, 2014

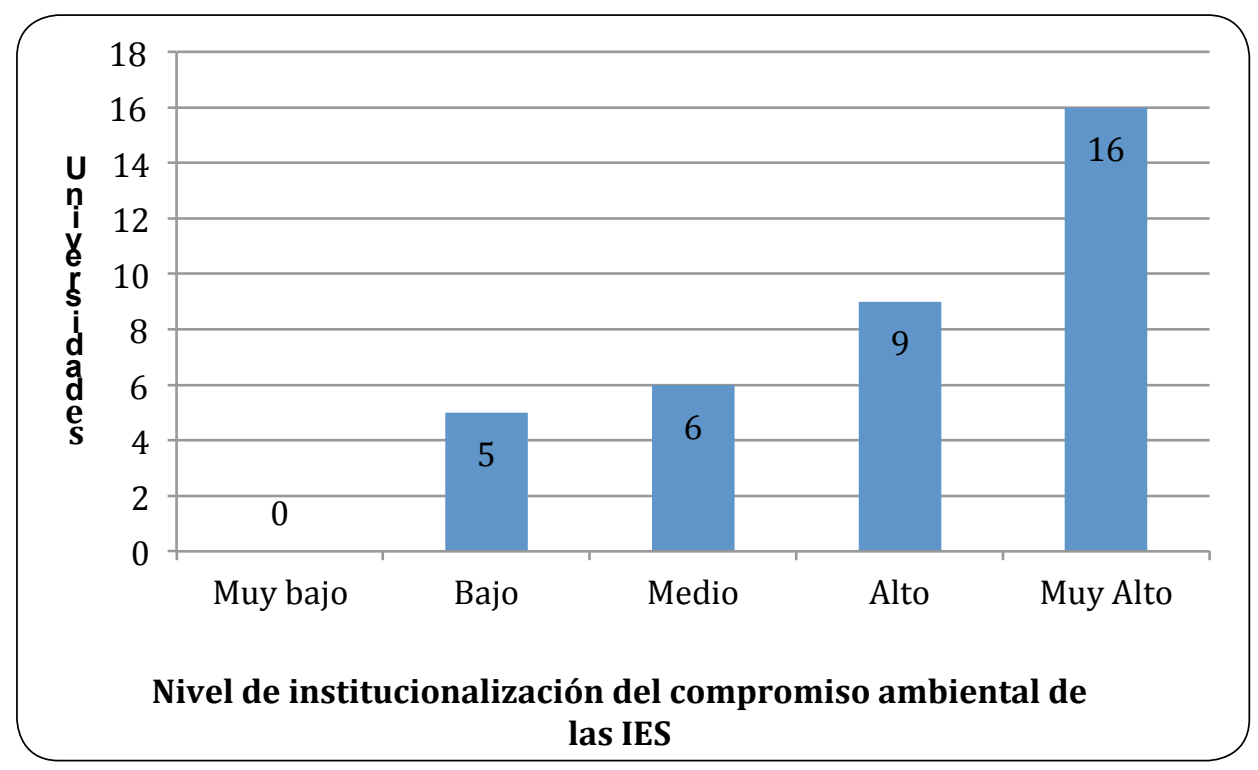

Fuente: Elaboración propia. 
Como se muestra en este gráfico, la mayor concentración de IES (16) se encuentra en el nivel muy alto; le siguen, en el nivel alto de institucionalización del compromiso ambiental, nueve centros educativos; en nivel medio se encuentran seis Universidades y en el nivel bajo apenas cinco. Es de resaltar que ninguna de las 36 Universidades que participaron en el estudio se ubica en el nivel muy bajo.

Estos resultados generales del diagnóstico señalan un nivel de institucionalización del compromiso ambiental de las universidades un poco más alto del que se esperaba con base en el conocimiento directo que los integrantes del equipo de investigación tienen sobre el estado actual del proceso, el cual vienen siguiendo muy de cerca desde hace dos décadas. Según esta percepción, compartida por otros expertos en el tema, el nivel actual del compromiso ambiental de las Universidades colombianas, en su conjunto, se encontraría en un nivel medio, dentro de la escala construida para esta investigación.

Lo que más sorprende de los resultados que se muestran en el gráfico anterior es el gran número de universidades (16) que, por sus respuestas, se ubican en el nivel superior de la escala. Para el equipo de investigación UAS (conformado por 6 investigadores especializados en el campo ambiental universitario y con participación activa en redes como la RCFAy RAUS), las instituciones de educación superior del país más avanzadas en su compromiso ambiental se ubican en el nivel alto, pero no en el nivel muy alto. Además, estas universidades no deben ser muchas. La mayoría de las universidades del país estarían en un nivel medio.

Una parte de la explicación para esta diferencia entre los resultados de la encuesta y el conocimiento directo (histórico y empírico) que se tiene de la situación estudiada, es el carácter básico y general de las preguntas que se plantearon a las universidades. Otra razón puede ser que, hasta ahora, los resultados surgen simplemente de las respuestas positivas, sin ninguna explicación odatos adicionales (producto de una verificación). La tercera y muy probable causa, como se anticipó desde el diseño de la metodología, es que la muestra al final obtenida en este estudio está necesariamente ubicada hacia arriba de la escala, presuntamente por ser Universidades que respondieron voluntariamente y que tenían procesos ya iniciados.

Las universidades con un nivel más alto de institucionalización de su compromiso ambiental tienden a participar en este tipo de encuestas, mientras que no lo hacen aquellas situadas en los niveles más bajos, por lo cual se esperaría que, si se tuviera la participación de todo o la mayoría del universo de este tipo de centros de educación superior, podrían presentarse unos puntajes inferiores a los datos estadísticos obtenidos. Con base en estos razonamientos, se pueden entender los datos que se presentan como una medición del nivel alcanzado por el grupo de universidades que más han avanzado en la institucionalización de su compromiso ambiental, pero no por el conjunto de este tipo de IES en Colombia.

El que un buen número de universidades se ubiquen en el nivel más alto de la escala y algunas obtengan el puntaje máximo posible se explica también por el carácter básico (e incluso exploratorio) de la encuesta y el reducido número de preguntas, así como la no inclusión de un proceso de verificación. En una escala mejorada, que se construya incluyendo preguntas adicionales, mucho más específicas y con un proceso de verificación y seguimiento, seguramente, pocas o ninguna se ubicarían en el nivel más alto de la misma.

En la siguiente fase de esta investigación se procederá a contrastar las respuestas afirmativas con la información, los textos y los documentos que las soportan. Además, se evaluará su pertinencia, su aprobación formal y su publicación en páginas web. Con base en esos criterios se construirá una nueva escala de medición de los niveles de institucionalización 
del compromiso ambiental de las universidades y lo más probable es que, con la aplicación de la verificación y la nueva escala de medición, los resultados del diagnóstico estén más próximos a los presupuestados con base en el conocimiento directo que se tiene sobre un proceso en el que se participa directamente y se estudia desde hace varios años, e incluso décadas.

\section{Ámbitos de acción.}

Además del nivel general de institucionalización del compromiso ambiental de las 36 Universidades colombianas que participaron, interesa conocer los resultados del diagnóstico para cada uno de los cinco ámbitos de acción en los que se organizaron las preguntas.

Gráfico 3. Puntaje promedio para cada ámbito de institucionalización del compromiso ambiental, 2014

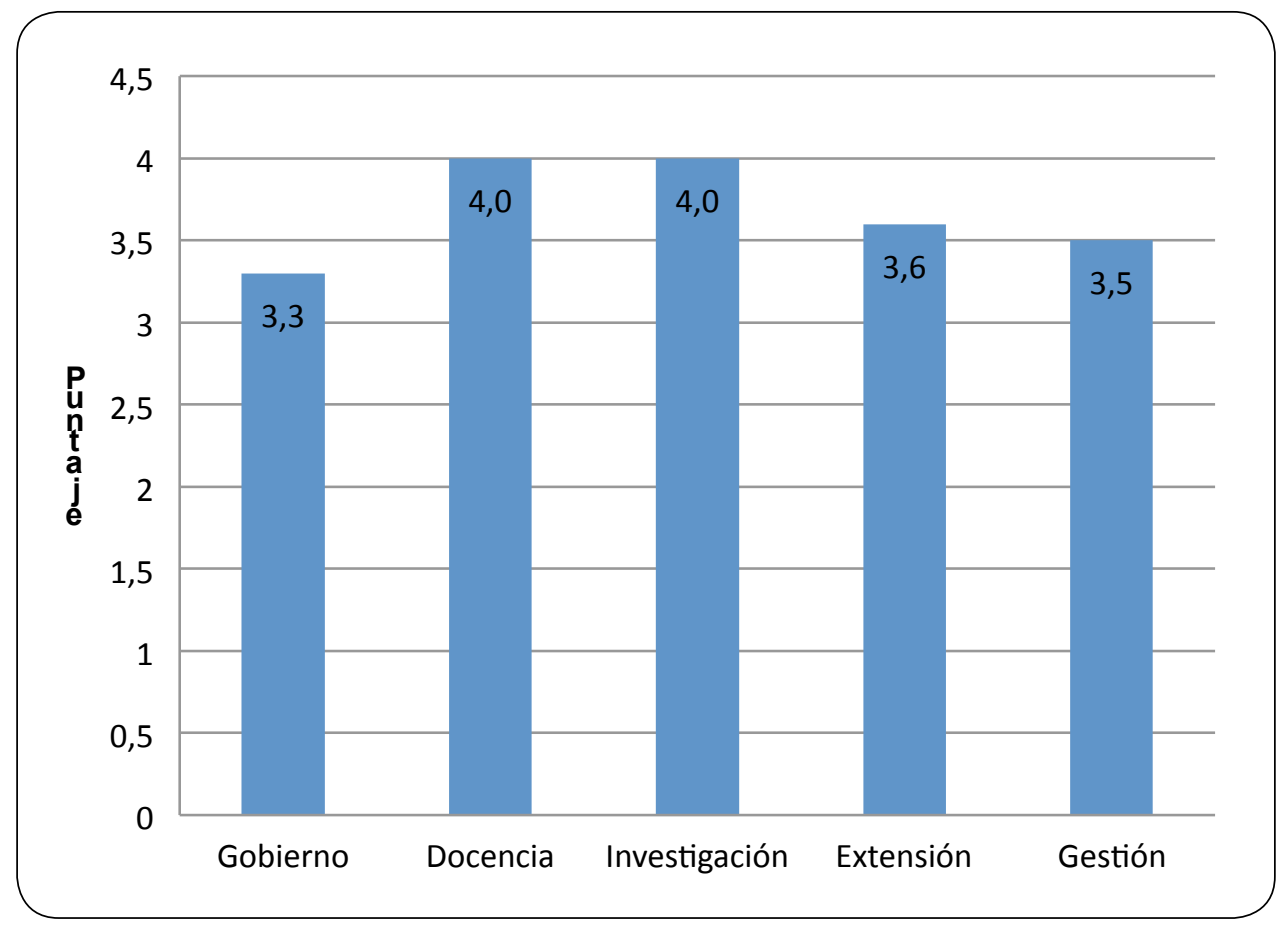

Fuente: Elaboración propia.

Como se puede observar en este gráfico, en una escala con un valor máximo de 5,0, el nivel más alto de avance promedio de las universidades en su compromiso ambiental se registra en los ámbitos de la docencia y la investigación, con un mismo valor de 4,0. Las posiciones siguientes son para la extensión $(3,6)$ y la gestión $(3,5)$. El ámbito del gobierno universitario en materia ambiental es el que tiene una calificación promedio menor $(3,3)$.

Lo primero que se evidencia en estos resultados, es que son los ámbitos correspon- dientes a dos de las tres clásicas funciones sustantivas de las universidades (investigación y docencia) los que presentan los promedios más altos. Se observa también que los ámbitos que más recientemente han asumido las universidades en el campo del ambiente y la sustentabilidad (gobierno y gestión), obtienen los puntajes promedios más bajos. La función académica de extensión o proyección social se ubica en un nivel intermedio.

Indiscutiblemente estos resultados son bastante consistentes, pues la docencia y la in- 
vestigación son los ámbitos de acción en los que las Universidades colombianas han venido incorporando los temas ambientales desde hace más de medio siglo, como se registra en los diagnósticos y estudios previos reseñados. En cambio, es apenas desde los años noventa que las instituciones de educación superior asumieron su responsabilidad en la gestión de sus campus (Sáenz, 2007, p. 259) y sólo más recientemente en sus decisiones de gobierno.

La coherencia de los resultados con el proceso histórico bien conocido de las instituciones que participan desde hace bastante tiempo en las redes universitarias ambientales del país, genera una mayor confianza en la capacidad de la escala utilizada para medir la institucionalización del compromiso ambiental de las universidades. En este aspecto, los resultados del diagnóstico coinciden ampliamente con el conocimiento directo que tiene el equipo de investigación sobre la situación actual del proceso.

\section{Preguntas de evaluación.}

Además de conocer el nivel de institucionalización del compromiso ambiental de las universidades en general y por cada uno de los ámbitos de acción, la encuesta permite medir aspectos aún más específicos de la puesta en práctica de dicho compromiso. En el Gráfico 4 se presenta el número de Universidades que respondieron afirmativamente cada una de las 25 preguntas básicas del cuestionario utilizado.

Estos datos son importantes porque permiten evaluar, al mismo tiempo, dos aspectos de gran interés para el diagnóstico: en primera instancia, conocer con mayor detalle el estado actual del proceso de institucionalización del compromiso ambiental de las Universidades colombianas; segundo, permiten valorar la utilidad de las preguntas específicas de evaluación empleadas para medir los niveles alcanzados en el avance de este proceso.

\section{Gráfico 4. Número de Universidades colombianas que responden de manera positiva a cada una de las 25 preguntas, 2014}

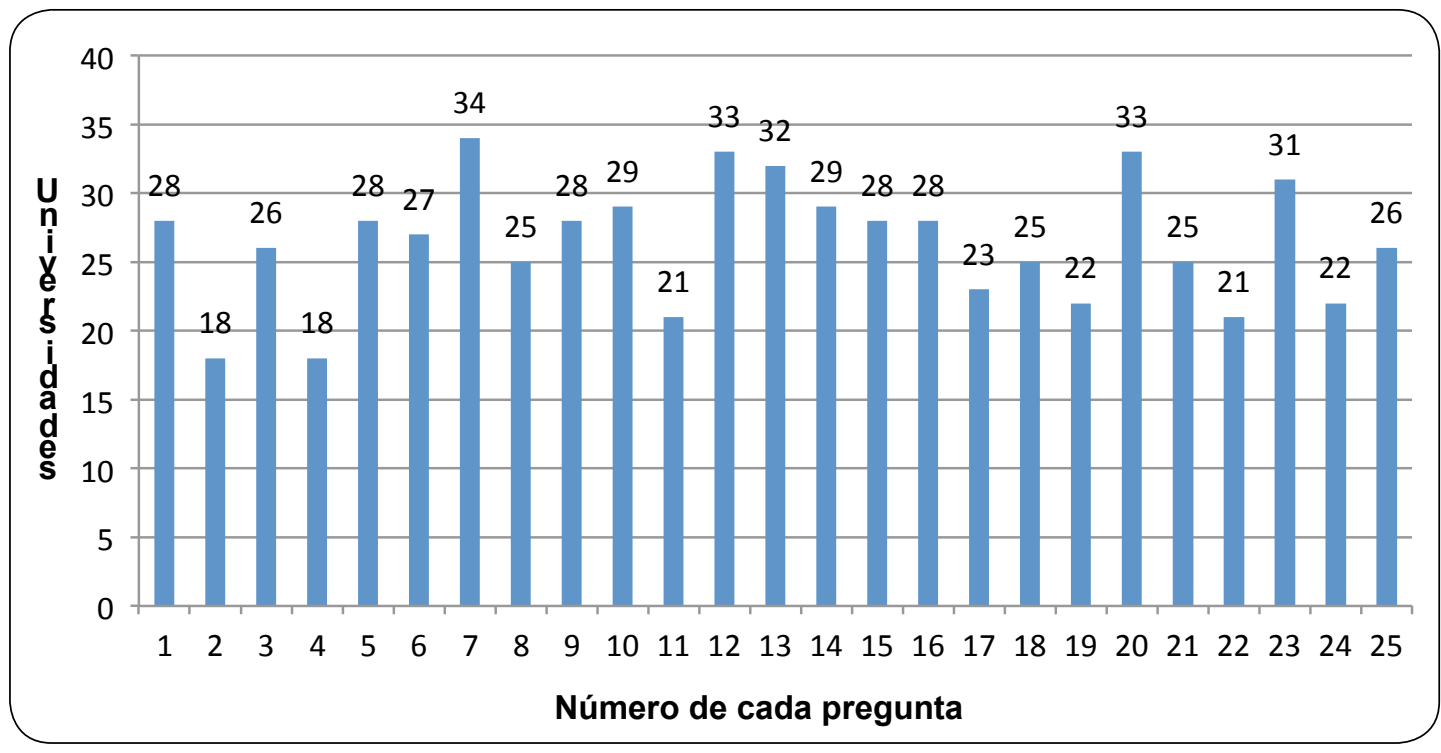

Fuente: Elaboración propia.

Gracias al gráfico, se observa que, en general, todos los niveles de respuesta afirmativa son altos: en todas las preguntas de la encuesta, las respuestas positivas fueron siempre superiores al $50 \%$, y el rango de respuestas positivas está entre $18(51,4 \%)$ y $34(94,4 \%)$. 
Estos resultados específicos son totalmente coherentes con los anteriores, en los que, con base en datos mucho más agregados, se llegaba a la misma conclusión: en general, son altos o muy altos los niveles alcanzados por las Universidades colombianas en la institucionalización del compromiso ambiental.

El valor mínimo (18) de respuestas afirmativas se obtuvo sólo en dos preguntas ( 2 y 4$)$ correspondientes al ámbito de gobierno. Como se puede ver en la Tabla 1, estas dos preguntas se refieren, respectivamente, a la existencia de una oficina o unidad administrativa especial responsable de los temas ambientales en la universidad y a la asignación de un presupuesto específico para su operación. El hecho de que coincidan las respuestas positivas para las dos preguntas, permite inferir que en las 18 Universidades que afirman tener dicha unidad administrativa especial, esta cuenta con presupuesto propio.

Es notable que este resultado estadístico de la encuesta sea coherente con el análisis que se hizo anteriormente sobre los ámbitos de acción, en el que el ámbito de gobierno obtuvo la menor calificación promedio en el conjunto de Universidades que respondieron la encuesta. Además, el hecho de que este resultado coincida con el conocimiento directo que tiene el equipo de investigación sobre la situación estudiada, refuerza la confianza que se puede tener en la capacidad de la encuesta para describir y medir los avances en el proceso de institucionalización del compromiso ambiental de las instituciones de educación superior.

En el otro extremo de las frecuencias presentadas en el Gráfico 4, la única pregunta que 34 de las 36 Universidades respondieron positivamente, fue la número 7 . Con ella se quería saber si se incorpora en algunos de los currículos académicos de las distintas carreras la perspectiva de la sustentabilidad ambiental y la respuesta es afirmativa en el $94,4 \%$ de los casos. Este resultado también es coherente por varias razones: la primera es el hecho histórico, ya re- señado en los antecedentes de la investigación, de que el proceso de incorporación de la dimensión ambiental en la educación superior en Colombia comenzó desde hace seis décadas, en el ámbito de la docencia o la formación ambiental. El eje principal de este proceso siempre ha sido, hasta ahora, la creación de nuevos programas de formación superior, a nivel de pregrado y posgrado, especializados en temas ambientales y de sustentabilidad.

Sumado a esto, se debe tener en cuenta que la pregunta es de carácter muy amplio porque se refiere sólo a algunos de los currículos académicos de las distintas carreras o programas que ofrecen las universidades. Lógicamente, era de esperar, como así ha sucedido, que casi todas las Universidades respondieran afirmativamente a esta pregunta. Con seguridad, las respuestas afirmativas habrían sido muchas menos si la pregunta implicara a todos los programas académicos de cada una de las instituciones que participaron en la encuesta.

Se pueden hacer observaciones muy similares referentes a la muy alta respuesta afirmativa $(91,6 \%)$ que obtuvo la pregunta 12 , la segunda en el orden de frecuencias que se puede apreciar en el Gráfico 4. El resultado también es coherente, porque fue precisamente el ámbito de investigación el que obtuvo la calificación más alta entre los cinco considerados. Igualmente coincide con el conocimiento empírico previo pues de todos es conocido que la mayoría de las Universidades colombianas cuenta con algún grupo de investigación específico, formalmente constituido, sobre ambiente o sustentabilidad.

La tercera pregunta, en el orden de frecuencias que se está analizando, fue la número 20 , referente a la participación de las Universidades en redes nacionales o internacionales sobre ambiente y sustentabilidad. Las respuestas afirmativas a esta pregunta fueron muy altas (33 de 36), tal como se esperaba, pues Colombia era en el 2014 el único país de América Latina y el Caribe que contaba con dos Redes Universitarias 
Ambientales (RCFA y RAUS) y la Red Colombiana de Formación Ambiental es la más antigua de la región, pues ha trabajado de manera ininterrumpida desde la década de los ochenta.

Volviendo la mirada a la encuesta empleada para este diagnóstico, así como los resultados, interesa también evaluar el instrumento que permitió la recolección de la información. Al respecto, ya se han señalado varios indicios favorables a la confiabilidad de la encuesta para medir bien el nivel de institucionalización del compromiso ambiental de las universidades, pues casi todos los resultados que se obtienen de su aplicación están en línea con el conocimiento previo, alcanzado por otros medios, que se tiene sobre este proceso.

Ya se ha señalado que la única excepción es el nivel promedio general encontrado, que es comprensiblemente un poco más alto que el esperado porque del grupo de Universidades que respondieron el formulario, hay una alta presencia de las instituciones que tienen mayor avance en su compromiso ambiental, en su mayoría integrantes de las redes universitarias ambientales que existen en Colombia (RCFA y RAUS).

Un último buen indicio sobre la calidad de la encuesta elaborada para este estudio es el hecho de que ninguna de las preguntas obtuvo el $100 \%$ de respuestas positivas o el $0 \%$ de respuestas negativas por parte de las 36 Universidades que participaron en este estudio. Por lo tanto, todos los criterios indagados a través de las preguntas, son útiles para establecer diferencias significativas, entre las universidades, con respecto al nivel que han alcanzado de institucionalización del compromiso ambiental. Este mismo resultado se obtuvo en el Proyecto RISU (Benayas et al., 2014), del cual se tomaron las preguntas.

\section{Consideraciones Finales}

Al comparar la presente investigación con los diagnósticos que la antecedieron en Co- lombia, se ha de resaltar el hecho de que con esta, se obtiene por primera vez una medición cuantitativa del nivel de avance alcanzado por las Universidades en la institucionalización de su compromiso ambiental. Todos los estudios previos se limitaron a informar el número de instituciones que respondían positivamente a las características o acciones por las que se preguntaba.

En cuanto se obtuvieron los primeros resultados del diagnóstico integral de cada una de las Universidades, se informó de manera confidencial a los respectivos centros educativos participantes. Esto permitió que cada institución pudiera compararse con los promedios nacionales, conocer el nivel de avance relativo que había alcanzado y definir acciones específicas para mejorar su desempeño y compromiso ambiental.

Es oportuno mencionar que uno de los problemas identificados por los investigadores es que algunas preguntas de la encuesta no fueron bien comprendidas por quienes la respondieron. Por esta razón, en las futuras fases del programa de investigación se estandarizará el protocolo de levantamiento de la información con el que deberán cumplir los funcionarios o representantes de las universidades que diligencien las encuestas; de esta manera, se buscará garantizar la idoneidad, legitimidad, y veracidad de los datos reportados. También se está pensando en confirmar, a través de un proceso de verificación in situ, la información suministrada. Se propone que en un tiempo cercano, las visitas de pares evaluadores se conviertan en un requisito para garantizar la confiabilidad de las respuestas y la información que se suministra.

Para superar la tendencia reconocida de que los resultados reflejan el nivel promedio de las universidades más avanzadas en su compromiso ambiental, en las siguientes fases de investigación se ampliará la muestra, de forma tal que refleje lo más cercano posible el universo 
de las IES. En el mediano plazo, se espera lograr que todas las Universidades del país respondan la encuesta o se diseñarán nuevas metodologías de trabajo que permitan conocer lo que cada institución hace en materia de ambiente y sustentabilidad.

En general, el Equipo UAS continuará desarrollando su programa de investigación con dos propósitos principales: obtener un conocimiento cada vez más amplio y preciso sobre el proceso de institucionalización del compromiso ambiental de las universidades, y construir mejores instrumentos para medir sus avances.

En concreto, la segunda fase de trabajo consiste en: i) Contrastar la respuestas afirmativas con la información, los textos y los documentos reportados; ii) Establecer la pertinencia de las respuestas afirmativas; iii) Verificar el carácter institucional de los documentos que las soportan; iv) Constatar su difusión pública a través de páginas web; y v) Construir una nueva escala de medición en la que se incluyan estos cuatro nuevos criterios de evaluación.

Todos estos avances en la segunda etapa de trabajo no implican abandonar el formulario utilizado sino aprovechar al máximo posible la información reportada por las Universidades. La encuesta con las 25 preguntas básicas se mantendrá por un tiempo, aplicando pequeños ajustes.

A mediano plazo se espera generar una encuesta con muchas más preguntas, que nuevamente se tomarán, en su mayor parte, del formulario del Proyecto RISU, ya puesto a prueba con un buen número de Universidades de Latinoamérica y el Caribe. En ese nuevo conjunto de preguntas se mantendrán las 25 básicas utilizada en la encuesta de 2014; las preguntas adicionales están orientadas a obtener un instrumento que permitirá medir con mayor detalle el nivel de institucionalización del compromiso ambiental de las Universidades colombianas.

\section{Nota}

1 Instituto Nacional de los Recursos Naturales Renovables y del Ambiente, disuelto en el año de 1993.

\section{Referencias}

Ángel, A. (1989). Programas Ambientales Universitarios. Diagnóstico. Bogotá: Instituto Colombiano para la Evaluación de la Educación -[ICFES]; Instituto Nacional de Recursos Naturales y Ambiente [INDERENA].

Benayas, J. Alba, D. Justel, A. Basterra. I. Guerra, A.F. Figueiredo, M., et al. (2014) Proyecto RISU. Definición de Indicadores para la evaluación de las políticas de sustentabilidad en universidades latinoamericanas. Madrid: Universidad Autónoma de Madrid; Alianza de Redes Iberoamericanas de Universidades por la Sustentabilidad y el Ambiente.

Bravo, M. T. (junio, 2005). Origeny desarrollode la Investigación en Educación Ambiental en México. Ponencia presentada en el "II Coloquio de Educación Ambiental UPN", México, Universidad Nacional Autónoma de México.

Cárdenas, J. M. (2014). Matriz de Indicadores de Incorporación de la Dimensión Ambiental en Universidades. Lima: Red Ambiental Interuniversitaria.

Centro Internacional de Formación en Ciencias Ambientales -[CIFCA]. (1978). Panorama de los Estudios Superiores Medioambientales en América Latina. En, La Formación Ambiental en América Latina (Vol 8, Cuadernos del CIFCA, pp. 55139). Madrid: Autor.

Equipo Universidad, Ambiente y Sustentabilidad -[UAS]. (2014a). Encuesta para el 
Diagnóstico de la Institucionalización del Compromiso Ambiental en las Universidades Colombianas. 2014 - 2015. Bogotá: Autor.

Equipo Universidad, Ambiente y Sustentabilidad -[UAS]. (2014b). Curso Taller Institucionalización del Compromiso Ambiental en las Universidades. Bogotá: Autor.

Hernandez, R. (2014). Metodología de la Investigación. México D.F: Mc Graw Hill.

Ministerio de Educación Nacional -[MEN]. (2014) Estadísticas de Educación Superior. Bogotá: Ministerio de Educación Nacional.

Organización de las Naciones Unidas para la Educación, la Ciencia y la Cultura [UNESCO]., Programa de las Naciones Unidas para el Medio Ambiente -[PNUMA]., \& Instituto Colombiano para la Evaluación de la Educación -[ICFES]. (1988). Universidad y Medio Ambiente en América Latina. Seminario de Bogotá. México. D.F.

Programa de las Naciones Unidas para el Medio Ambiente -[PNUMA]. (1985). La Incorporación de la Dimensión Ambiental en América Latina y el Caribe. Primer Seminario sobre "Universidad y Medio Ambiente para América Latina y el Caribe", México.

Programa de las Naciones Unidas para el Medio Ambiente -[PNUMA]. (2014). Decisión del XIX Foro de Ministros de Medio Ambiente para América Latina y el Caribe sobre Educación Ambiental para el Desarrollo Sostenible. Recuperado de http:// www.pnuma.org/educamb/documentos/foro/Final $\% 20$ decision $\% 20$ Edu $\% 20$ Amb\%20SP.pdf

Román, Y. (2016). Sistema Ambiental Universitario: Modelo integrado de Gestión para la Inclusión de la Dimensión Ambiental y Urbana en la Educación Superior. Bogotá: Universidad Piloto de Colombia.

Sáenz, O. (Ed.). (octubre, 2007). Memorias del Cuarto Seminario Internacional Universidad y Ambiente. Bogotá, Universidad de Ciencias Aplicadas y Ambientales.

Sáenz, O. (2012). La Formación Ambiental Superior. 1948-1991. Bogotá: Universidad de Ciencias Aplicadas y Ambientales y Red Colombiana de Formación Ambiental.

Sáenz,O.(2014).Panorama dela Sustentabilidad en las Universidades de América Latina y El Caribe. En A. Ruscheinsky, A. Guerra, M. Figueiredo, P. Silva, V. Lima \& W. Carvalho (Coords.), Ambientalização nas Instituições de Educação Superior no Brasil: Caminhos Trilhados, Desafios e Possibilidades (pp. 23-38). São Paulo: Universidade de São Paulo.

Sáenz, O. (2015a). Diagnósticos Nacionales sobre la Inclusión de Consideraciones Ambientales en las Universidades de América Latina y El Caribe. Revista iberoamericana Universitaria en Ambiente, Sociedad y Sustentabilidad, 1(1), 13-36.

Sáenz, O. (Ed.). (2015b). Universidades y Sostenibilidad en América Latina $y$ el Caribe. Bogotá: Universidad de Ciencias Aplicadas y Ambientales; Red Colombiana de Formación Ambiental.

Sáenz, O. (en prensa). Diagnósticos Regionales sobre la Inclusión de Consideraciones Ambientales en las Universidades de América Latina y El Caribe. En preparación.

Sánchez, V. (1984). Estudio sobre la incorporación de la Formación Ambiental a la Educación Superior en México (Informe de Investigación). México: El Colegio de México. Programa Desarrollo y Medio Ambiente. 


\section{Potencial conflicto de intereses}

Todos los autores de este artículo son profesores - investigadores vinculados a instituciones de educación superior que participaron en el diagnóstico de la institucionalización del compromiso de las Universidades colombianas que aquí se presenta. Sin embargo, como se ha visto, en el estudio no se hace ninguna referencia a universidades específicas, por lo que no existe interés en influir sobre los resultados de sus evaluaciones. Los resultados particulares de cada Universidad se entregaron en el mes de diciembre de 2014 a sus rectores o representantes y algunos de ellos han expresado su utilidad para reforzar y mejorar su trabajo en campo del ambiente y la sustentabilidad.

\section{Fuentes de financiación}

Para la ejecución de esta investigación sólo se requirió del tiempo que le dedicaron los investigadores durante el año 2014. La mayor parte de este tiempo hacía parte de sus respectivas jornadas laborales, por lo que la financiación se limitó a este aspecto del trabajo y estuvo a cargo de las respectivas Universidades: la Universidad de Ciencias Aplicadas y Ambientales, la Universidad Sergio Arboleda, la Universidad Libre y la Universidad Distrital Francisco José de Caldas. 
